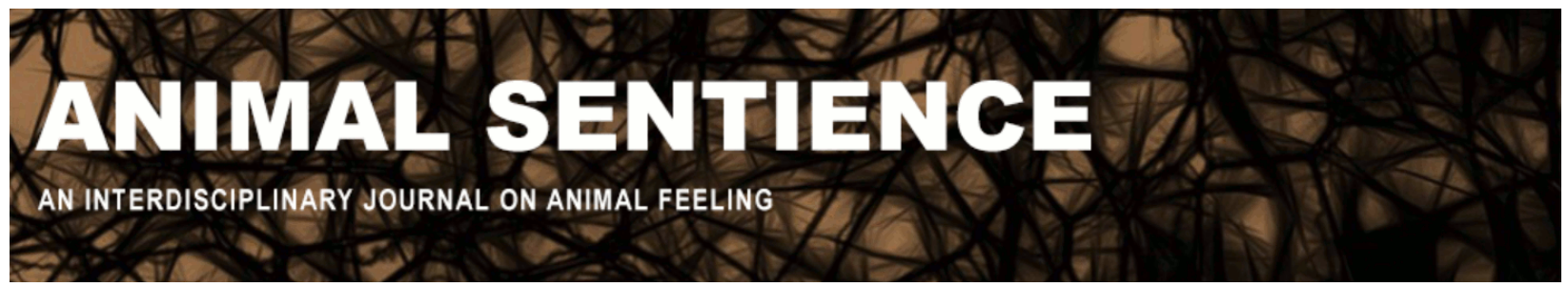

Adolphs, Ralph (2018) What would we like to know by imaging the brains of dogs?. Animal Sentience 22(14)

DOI: $10.51291 / 2377-7478.1344$

Date of submission: 2018-06-08

Date of acceptance: 2018-06-12

(c)

This article has appeared in the journal Animal

Sentience, a peer-reviewed journal on animal

cognition and feeling. It has been made open access,

free for all, by WellBeing International and deposited

in the WBI Studies Repository. For more information,

please contact

wbisr-info@wellbeingintl.org.

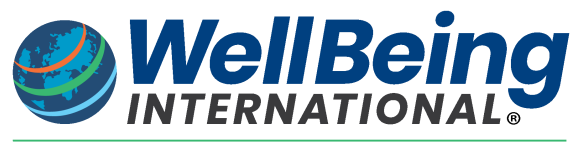

SOLUTIONS FOR PEOPLE, ANIMALS AND ENVIRONMENT 


\title{
What would we like to know by imaging the brains of dogs?
}

Commentary on Cook et al. on Dog Jealousy

\author{
Ralph Adolphs \\ California Institute of Technology
}

\begin{abstract}
Using fMRI to study emotions in animals is important, fascinating, and fraught with methodological and conceptual problems. Cook et al. are doing it, and there is no question that they and others will be doing it better and better as time goes on. Where will this lead us? What could $\mathrm{fMRI}$ in principle tell us about the minds of nonhuman animals?
\end{abstract}

Ralph Adolphs is a social neuroscientist who studies emotions in humans. Bren Professor of Psychology, Neuroscience, and Biology at California Institute of Technology; and Allen V. C. Davis and Lenabelle Davis Leadership Chair and Director, Caltech Brain Imaging Center, Adolphs is the co-author of the new book, The neuroscience of emotion: A new synthesis, Princeton University Press, 2018. Website

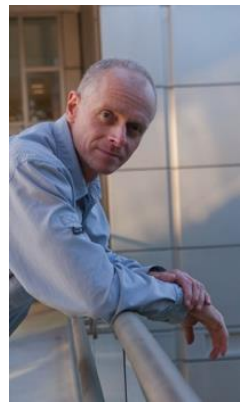

Neuroimaging of dogs is difficult. Cook et al. (2018) continue their intriguing line of work using $\mathrm{fMRI}$ in dogs. The findings are fascinating and important, and the attention to the well-being of the animal participants is impressive. The studies also have a number of strong caveats that currently limit clear conclusions (as the authors themselves are well aware). For one, there are strong technical limitations, mostly with respect to statistical power. For instance, the correlations shown in Figure 2A cannot be considered statistically meaningful (Schönbrodt and Perugini, 2013). But there is also a concern about interpretation of the findings, even if one takes them at face value (as I do here for the sake of the argument).

Foremost is the problem of "reverse inference": inferring a cognitive process from brain activations alone (Poldrack, 2011). There are two ways to counter this problem. One is to have a very large number of prior imaging results that provide high specificity to the activations observed. This is not the case here, although the activations do correspond to those observed in some human studies of jealousy - but they also correspond to activations seen in many more studies that in no way involve jealousy. As Cook et al. note, "amygdala activation should not be equated with specific emotions; it should be more broadly interpreted...."

A second counter is more relevant here: it is not only the brain activations that are used for the inference. The dogs are put into situations that are functionally reasonable to evoke jealousy, and in fact behaviors consistent with jealousy have been observed when dogs were put into similar situations outside the scanner. Putting all these together, one can make a qualified interpretation that the dogs in the scanner were in a functional state of dog-jealousy or something close to it, while still leaving open plenty of debate about exactly how to define that. Most studies of animal behaviors that have close homologs in human behavior have focused on 
trying to make this interpretation airtight and eliminating less specific or confounding explanations. But that is not the most interesting aspect of the present study.

Peeking into the mind of a dog. Suppose instead that it is the year 2040 and OpenfMRI for dogs has repositories of thousands of fMRI studies on dogs, typical fMRI studies in dogs and other animals have vast statistical power, and airtight behavioral protocols have been designed to unambiguously attribute dog-jealousy to dogs (or at least, everybody has reached a consensual agreement on this issue). All the above problems have been surmounted. What can we conclude?

Let's start with a simple question: what kind of a variable is "jealousy" supposed to be? It causes behavior, a measured variable, and so we can think of jealousy as a latent variable inferred from, and causing, behavior and autonomic responses. The present study doesn't measure behavior; it measures brain activity. Does jealousy cause the observed brain activity, just as it causes the behavior? Or is it the other way around: the brain activity causes jealousy? Or are brain function and jealousy related in some other way? The study only shows us correlations, but we would like to infer a relation more specific than that.

Cook et al. view their amygdala activation more or less like a biomarker: it provides useful predictive information about the temperament of a dog. If that were all there was to it, we would ask what added value fMRI gives us, above and beyond just knowing the behavioral history of a dog, or perhaps its genetics. Given the cost involved and the difficulties in quantifying and interpreting the data, $\mathrm{fMRI}$ seems like a terrible candidate for a biomarker. The interest in dog $\mathrm{fMRI}$ - which is patently there - must lie in the possibility that it gives us a peek inside the dog's mind. It must tell us something that behavior could not.

I think the strong assumption is that fMRI actually shows us the causal mechanisms that are the physical realization of the functional state of jealousy. And if you believe that conscious experiences of jealousy supervene on the physical realization of the functionally defined state of jealousy, then fMRI would show you the conscious experience of jealousy (or that on which it directly depends). This would have several implications (very roughly):

1. The brain activity that we can measure with $\mathrm{fMRI}$ would completely determine the conscious experience of jealousy; so we could unambiguously read-out whether or not a dog felt jealous from the pattern of brain activations;

2. If we manipulated the brain activity, we could change the conscious experience of jealousy. Drugs for jealousy could be tested in this way in animal models, for instance.

If valid, these points would have revolutionary consequences for the use of $\mathrm{fMRI}$ data. The ethical treatment of animals would have clear data from which to reason. Animal studies of drugs to treat depression would, contra recent calls to the contrary (LeDoux and Pine, 2016), be valid models for treating depression in humans as well (and for treating depressed animals, for that matter).

The future. So the prescription for a neuroscience of animal emotions might proceed in three stages. First, we need much better neuroscience methods with better statistical reliability and reproducibility, a necessary step that seems eminently surmountable (at least by 2040). Second, 
we need clearer, more detailed, and ecologically valid functional definitions of emotions, and protocols to test them. The difficulty of this step will no doubt vary by emotion: for some, like fear, we are essentially already there; for jealousy, not yet; for awe, probably never will be. As my colleague Dean Mobbs (2018) points out in his commentary, in the case of dogs, we would need to pay close attention not just to how dogs interact with one another, but also to how they interact with humans: many dog emotions have a function in social communication with (or, less kindly but perhaps more accurately, instrumental manipulation of) their owners. Third, we then need to link the reliable neuroscience findings with the ecologically valid functional operationalizations of the emotion and with reports or direct experiences of feeling the emotion (from studies in humans).

It is important to note that these three ingredients of an emotion (functional definition, brain state, and conscious experience) all cohere to some extent, but will also vary to some extent across species. Dog jealousy does not cause the exact same behaviors as in humans; that's why dog ecology needs to be taken into account. Dog brains are not human brains, and some differences in activation patterns must obtain; the multiple realizability of the functionally defined emotion state will allow further variability. And being a jealous dog of course doesn't feel like being a jealous person. Yet if the functional definition and fMRI results are sufficiently similar to what we can make sense of in humans, fMRI will turn out to be an indispensable additional measure to enrich our understanding of the conscious experiences of animals.

\section{References}

Cook, P., Prichard, A., Spivak, M., and Berns, G. S. (2018). Jealousy in dogs? Evidence from brain imaging. Animal Sentience 22(1).

LeDoux, J., and Pine, D. (2016). Using neuroscience to help understand fear and anxiety: A twosystem framework. American Journal of Psychiatry 173: 1083-1093.

Mobbs, D. (2018). What can the social emotions of dogs teach us about human emotions? Animal Sentience 22(5).

Poldrack, R. A. (2011). Inferring mental states from neuroimaging data: From reverse inference to large-scale decoding. Neuron 72: 692-697.

Schönbrodt, F. D., and Perugini, M. (2013). At what sample size do correlations stabilize? Journal of Research in Personality 47: 609-612. 


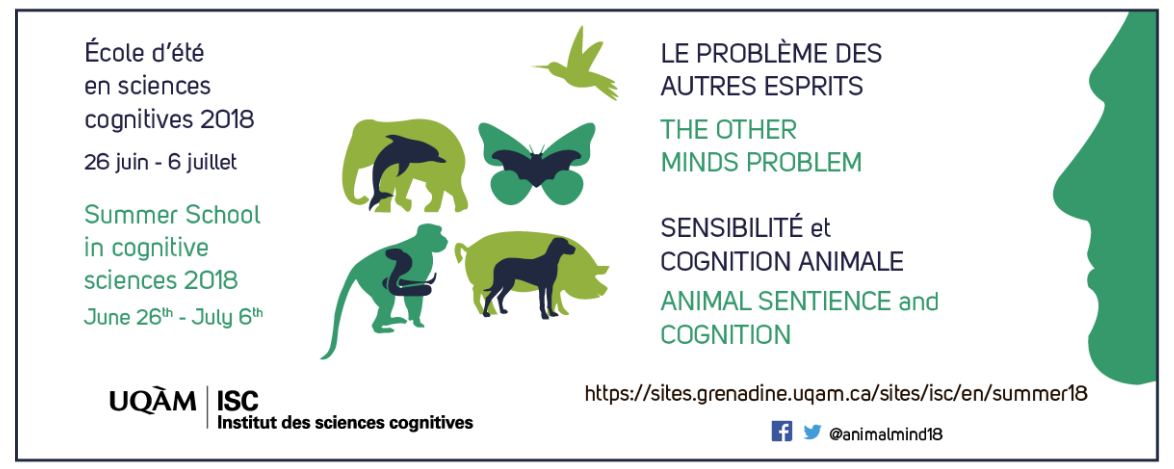

ISC 2018 Summer School in Cognitive Sciences June 26 - July 6, 2018 Montreal (Canada)

\section{The Other Minds Problem: Animal Sentience and Cognition}

Overview. Since Descartes, philosophers know there is no way to know for sure what — or whether - others feel (not even if they tell you). Science, however, is not about certainty but about probability and evidence. The 7.5 billion individual members of the human species can tell us what they are feeling. But there are 9 million other species on the planet (20 quintillion individuals), from elephants to jellyfish, with which humans share biological and cognitive ancestry, but not one other species can speak: Which of them can feel — and what do they feel? Their human spokespersons - the comparative psychologists, ethologists, evolutionists, and cognitive neurobiologists who are the world's leading experts in "mindreading" other species - will provide a sweeping panorama of what it feels like to be an elephant, ape, whale, cow, pig, dog, chicken, bat, fish, lizard, lobster, snail: This growing body of facts about nonhuman sentience has profound implications not only for our understanding of human cognition, but for our treatment of other sentient species.

Gregory Berns: Decoding the Dog's Mind with Awake Neuroimaging Gordon Burghardt: Probing the Umwelt of Reptiles

Jon Sakata: Audience Effects on Communication Signals

PANEL 1: Reptiles, Birds and Mammals

WORKSHOP 1: Kristin Andrews: The "Other" Problems: Mind, Behavior, and Agency

Sarah Brosnan: How Do Primates Feel About Their Social Partners? Alexander Ophir: The Cognitive Ecology of Monogamy

Michael Hendricks: Integrating Action and Perception in a Small Nervous System

PANEL 2: Primates, Voles and Worms

WORKSHOP 2: Jonathan Birch: Animal Sentience and the

Precautionary Principle

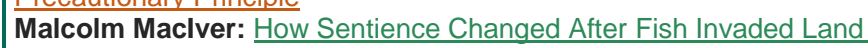
385 Million Years Ago

Sarah Woolley: Neural Mechanisms of Preference in Female

\section{Songbird}

Simon Reader: Animal Social Learning: Implications for

Understanding Others

PANEL 3: Sea to Land to Air

WORKSHOP 3: Steven M. Wise: Nonhuman Personhood

Tomoko Ohyama: Action Selection in a Small Brain (Drosophila

Maggot)

Mike Ryan: "Crazy Love": Nonlinearity and Irrationality in Mate Choice

Louis Lefebvre: Animal Innovation: From Ecology to

Neurotransmitters

PANEL 4: Maggots, Frogs and Birds: Flexibility Evolving SPECIAL EVENT: Mario Cyr: Polar Bears

Colin Chapman: Why Do We Want to Think People Are Different?

Vladimir Pradosudov: Chickadee Spatial Cognition

Jonathan Balcombe: The Sentient World of Fishes

PANEL 5: Similarities and Differences

WORKSHOP 5 (part 1): Gary Comstock: A Cow's Concept of Her Future

WORKSHOP 5 (part 2): Jean-Jacques Kona-Boun: Physical and Mental Risks to Cattle and Horses in Rodeos
Joshua Plotnik: Thoughtful Trunks: Application of Elephant Cognition for Elephant Conservation

Lori Marino: Who Are Dolphins?

Larry Young: The Neurobiology of Social Bonding, Empathy and

Social Loss in Monogamous Voles

Panel 6: Mammals All, Great and Small

WORKSHOP 6: Lori Marino: The Inconvenient Truth About Thinking Chickens

Andrew Adamatzky: Slime Mould: Cognition Through Computation Frantisek Baluska \& Stefano Mancuso: What a Plant Knows and Perceives

Arthur Reber: A Novel Theory of the Origin of Mind: Conversations With a Caterpillar and a Bacterium

PANEL 7: Microbes, Molds and Plants

WORKSHOP 7: Suzanne Held \& Michael Mendl: Pig Cognition and Why It Matters

James Simmons: What Is It Like To Be A Bat?

Debbie Kelly: Spatial Cognition in Food-Storing

Steve Phelps: Social Cognition Across Species

PANEL 8: Social Space

WORKSHOP 8: To be announced

Lars Chittka: The Mind of the Bee

Reuven Dukas: Insect Emotions: Mechanisms and Evolutionary Biology

Adam Shriver: Do Human Lesion Studies Tell Us the Cortex is Required for Pain Experiences?

PANEL 9: The Invertebrate Mind

WORKSHOP 9: Delcianna Winders: Nonhuman Animals in Sport and Entertainment

Carel ten Cate: Avian Capacity for Categorization and Abstraction Jennifer Mather: Do Squid Have a Sense of Self?

Steve Chang: Neurobiology of Monkeys Thinking About Other Monkeys

PANEL 10: Others in Mind

WORKSHOP 10: The Legal Status of Sentient Nonhuman Species 